OPEN ACCESS

Edited by:

Marc Vooijs,

Maastricht University, Netherlands

Reviewed by:

Lucio Miele,

LSU Health Sciences Center New Orleans, United States

Sathish Kumar Mungamuri, Asian Institute of Gastroenterology,

India

*Correspondence:

Paolo Sportolett sporto/p@gmail.com

tThese first authors contributed equally to this article.

*These senior authors contributed equally to this article.

Specialty section: This article was submitted to Molecular and Cellular Oncology, a section of the journal Frontiers in Oncology

Received: 26 January 2018 Accepted: 23 March 2018

Published: 20 April 2018

Citation: Di lanni M, Baldoni S, Del Papa B, Aureli P, Dorillo E, De Falco F, Albi E,

Varasano E, Di Tommaso A, Giancola R, Accorsi P, Rotta G, Rompietti $C$, Silva Barcelos EC, Campese AF, Di Bartolomeo P, Screpanti I, Rosati E, Falzetti F and Sportoletti P (2018) NOTCH1 is Aberrantly Activated in Chronic Lymphocytic Leukemia Hematopoietic Stem Cells. Front. Oncol. 8:105 doi: 10.3389/fonc. 2018.00105

\section{NOTCH1 Is Aberrantly Activated in Chronic Lymphocytic Leukemia Hematopoietic Stem Cells}

\author{
Mauro Di lanni ${ }^{1,2+}$, Stefano Baldoni ${ }^{3 \dagger}$, Beatrice Del Papa ${ }^{4 \dagger}$, Patrizia Aureli ${ }^{4}$ Erica Dorillo ${ }^{4}$, \\ Filomena De Falco ${ }^{4}$, Elisa Albi ${ }^{4}$, Emanuela Varasano ${ }^{4}$, Ambra Di Tommaso ${ }^{3}$, Raffaella \\ Giancola ${ }^{2}$, Patrizia Accorsi ${ }^{2}$, Gianluca Rotta ${ }^{5}$, Chiara Rompietti ${ }^{4}$, Estevão Carlos Silva \\ Barcelos $^{4,6}$, Antonio Francesco Campese ${ }^{7}$, Paolo Di Bartolomeo ${ }^{2}$, Isabella Screpanti \\ Emanuela Rosati ${ }^{8}$, Franca Falzetti ${ }^{4 \neq}$ and Paolo Sportoletti ${ }^{4 *}$
}

\begin{abstract}
Department of Medicine and Aging Sciences, University of Chieti Pescara, Chieti, Italy, ${ }^{2}$ Department of Hematology, Transfusion Medicine and Biotechnologies, Ospedale Civile, Pescara, Italy, ${ }^{3}$ Department of Life, Health and Environmental Sciences, Hematology Section, University of L'Aquila, L'Aquila, Italy, ${ }^{4}$ Institute of Hematology-Centro di Ricerche EmatoOncologiche (CREO), University of Perugia, Perugia, Italy, ${ }^{5}$ BD Biosciences, San Jose, Italy, ${ }^{6}$ Universidade Federal do Espirito Santo, Vitória, Brazil, ${ }^{7}$ Department of Molecular Medicine, Sapienza, University of Rome, Rome, Italy, ${ }^{8}$ Department of Experimental Medicine, Biosciences and Medical Embriology Section, University of Perugia, Perugia, Italy
\end{abstract}

To investigate chronic lymphocytic leukemia (CLL)-initiating cells, we assessed NOTCH1 mutation/expression in hematopoietic stem cells (HSCs). In NOTCH1-mutated CLL, we detected subclonal mutations in 57\% CD34+/CD38- HSCs. NOTCH1 mutation was present in 66\% CD34+/CD38+ progenitor cells displaying an increased mutational burden compared to HSCs. Flow cytometric analysis revealed significantly higher $\mathrm{NOTCH} 1$ activation in CD34+/CD38- and CD34+/CD38+ cells from CLL patients, regardless $\mathrm{NOTCH} 1$ mutation compared to healthy donors. Activated NOTCH1 resulted in overexpression of the NOTCH1 target C-MYC. We conclude that activated NOTCH1 is an early event in CLL that may contribute to aberrant HSCs in this disease.

Keywords: notch signaling, chronic lymphocytic leukemia, hematopoietic stem cells, NOTCH1 mutation, CD34+ cells

\section{INTRODUCTION}

Chronic lymphocytic leukemia (CLL) is a mature B cell malignancy characterized by accumulation of clonal B cells in blood, bone marrow (BM), and lymphoid tissues. The search for CLL-initiating cells has never been successful. While several cell types have been suggested as giving rise to CLL, yet, there is no consensus as to its normal cellular counterpart (1). CLL cells have monoclonal immunoglobulin gene rearrangements, suggesting that lymphoid malignant stem cells originate after cells have committed to the lymphoid lineage. More recently, it has been reported that hematopoietic stem cells (HSCs) from CLL patients display the propensity to generate clonal B cells, suggesting the involvement of HSCs in lymphoid leukemogenesis (2).

In 2009, we first identified NOTCH1 mutations in CLL (3) and provided data on the adverse prognostic outcome associated with mutated NOTCH1 (4). More recently, independent studies confirmed the presence and the prognostic relevance of NOTCH1 mutations in CLL patients (5). All mutations resulted in NOTCH1 impaired degradation that led to NOTCH1 deregulated signaling, indicating that mutations could contribute to increase NOTCH activation in CLL $(6,7)$. Recently, analyses of peripheral blood CD34+CD19- cells and BM hematopoietic progenitors revealed 
NOTCH1 mutation in some CLL samples $(8,9)$. Conversely, others failed to demonstrate the presence of NOTCH1 mutations in circulating CD34+ cells from NOTCH1 mutated patients (10). In lymph node, CLL cells show NOTCH1 activation independent of mutation (11) and recent evidence have shown non-mutational NOTCH1 signaling with anti-apoptotic effects in peripheral blood CLL cells (12). However, the role of NOTCH1 signaling in the HSC compartment of CLL is still unknown.

\section{MATERIALS AND METHODS}

\section{Cell Separation and Flow Cytometry}

We collected $28 \mathrm{BM}$ samples including $21 \mathrm{CLL}$ patients (15 NOTCH1 mutated and 6 NOTCH1 wild type) and 7 healthy donors (HDs). BM and peripheral blood (PB) cells were collected under signed informed consent in accordance with Declaration of Helsinki and the Institutional Review Board of University of Perugia. Patient's characteristics are described in Table S1 in Supplementary Material. BM cells from 10 NOTCH1 mutated CLL were single and/or double-sorted into CD34+CD38- HSCs and CD34+CD38+ progenitor fraction containing myeloid and lymphoid progenitors. Briefly, BM mononuclear cells were separated by Ficoll-Hypaque density gradient centrifugation. Flow cytometric analysis and cell-sorting were performed using the following antibodies: PE anti-CD34 and PC5 anti-CD38 (Beckman Coulter), PerCP-Cy5.5 anti-CD34, FITC anti-CD19, PE-Cy7 anti-CD38, APC-H7 anti-CD10, V450 anti-CD3, and V500 anti-CD45 (BD Biosciences). NOTCH1 ICN on sorted populations was performed using PE anti-NOTCH1 (mN1A) (eBioscience). Cells were analyzed using a FACSCanto II and sorted using a FACS Aria III cell sorter (BD Biosciences).

\section{Direct Sanger Sequencing}

NOTCH1 gene mutational analysis was performed by directional sequencing of PCR fragments from genomic DNA. Primers and PCR conditions were as previously described (13).

\section{Allele-Specific PCR (AS-PCR), Droplet Digital PCR (ddPCR), RT-PCR}

Allele-specific PCR was performed accordingly to a previously published protocol (13). ddPCR NOTCH1 probes assays (dHsaCP2500500 and dHsaCP2500501 Bio-Rad) were used to determinate the allelic burden of NOTCH1 in sorted cells. The droplet generated included DNA, Notch 1 probes assays $(1 \times)$, and ddPCR Supermix $(2 \times)$ for Probes (no dUTP) (Bio-Rad). The mix was amplified by PCR and analyzed by QX200 Droplet Reader (Bio-Rad). Scatterplots depicting ddPCR results specifically for the NOTCH1 mutation assay. Real-time PCR analysis of c-MYC and Hes1 gene expression was performed in immunoselected CD34+ BM cells. RNA was extracted using RNeasy Plus Kits (Qiagen, Hilden, Germany), and cDNA was obtained using Prime Script RT Master Mix (Takara Bio, Dalian, China). Real-time qPCR was performed with PCR Master Mix Power SYBER Green (Applied Biosystem, Warrington, UK) using the 7900HT fast Real-Time PCR System (Applied Biosystem). The primers sequence were F:5'CTTCTCTCCGTCCTCGGATTCT-3' andR:5'-GAAGGTGATC
CAGACTCTGACCTT-3' for c-Myc, F:5'-AAGAAAGATAG CTCGCGGCAT-3' and R:5'-CCAGCACACTTGGGTCTGT-3' for Hes 1 and F:5' - ATGGGGAAGGTGAAGGTCG-3' and R:5' GGGGTCATTGATGGCAACAATA-3' for GAPDH. Relative fold change was normalized to GAPDH and calculated using the $2^{-\Delta \Delta \mathrm{Ct}}$ method.

\section{Western Blot Analysis}

Whole-cell lysates extracted from BM CD34+ cells $\left(5 \times 10^{5}\right)$ of CLL patients and HDs were analyzed by western blot using an anti-NOTCH1 antibody (clone bTAN20) able to detect the $300-\mathrm{kDa}$ inactive precursor (FL), the $120-\mathrm{kDa}$ transmembrane/ cytoplasmic/cytoplasmic (TM) subunit, and the active $100-\mathrm{kDa}$ intracellular domain. Whole-cell lysates $(3 \mu \mathrm{g})$ isolated from peripheral blood CD5+CD19+ CLL cells of NOTCH1 mutated patients were used as positive control.

\section{Statistical Analysis}

Statistical analyses were performed with GraphPad (GrapdhPad Software Inc., La Jolla, CA, USA). In the text, data are presented as mean $\pm \mathrm{SD}$ and statistical differences between mean values were evaluated using the Student's $t$-test and Mann-Whitney test.

\section{RESULTS}

\section{HSCs From CLL Patients Showed NOTCH1 Mutation}

The mean proportion of BM CD34+ cells before enrichment was $0.75 \pm 0.44 \%$. After the FACS sorting procedures, the mean purity of CD34+/CD38- cells was $94.58 \pm 3.52 \%$ and CD34+/ CD38+ cells were $98.12 \pm 1.34 \%$ (Figure 1A).

We initially analyzed the NOTCH1 mutational hotspot by Sanger sequencing. The CD34+/CD38- fraction did not contain NOTCH1-mutated cells. Then, a high sensitivity AS-PCR assay for the NOTCH1 mutation (13) indicated the presence of small HSCs mutated clones in 57\% CLL samples. Densitometric analysis revealed a mean $6.4 \pm 4.7 \%$ NOTCH1 mutant allelic burden. Moreover, we used a ddPCR assay to validate NOTCH1 mutational data in HSCs by a more quantitative method. We confirmed NOTCH1 mutations in two HSCs samples for which DNA was available that display an allelic ratio of 2.6 and $8.5 \%$, respectively (Figure 1B). Altogether, these data confirm that NOTCH1 mutation is an early event in CLL hematopoiesis in a fraction of patients. Additionally, we measured the NOTCH1 mutational burden along specific stages of HSC differentiation in NOTCH1-mutated CLL patients. The mean percentage of the mutant allele progressively increased from $6.4 \pm 4.7 \%$ in CD34+CD38- to $14.9 \pm 11.3 \%$ in $\mathrm{CD} 34+\mathrm{CD} 38+\mathrm{CD} 10+\mathrm{CD} 19+$ cells, $22.7 \pm 6.5 \%$ in CD34-CD38+CD10+CD19+ cells and $40.5 \pm 4.3 \%$ in neoplastic CD5+CD19+ cells (Figure 1C). The analysis of the rearrangement status of the IgH gene revealed in both NOTCH1 mutated and NOTCH1 unmutated CD34+CD38- HSCs the presence of a germline configuration in the half of the samples while the other $50 \%$ showed a clonal VDJ. 


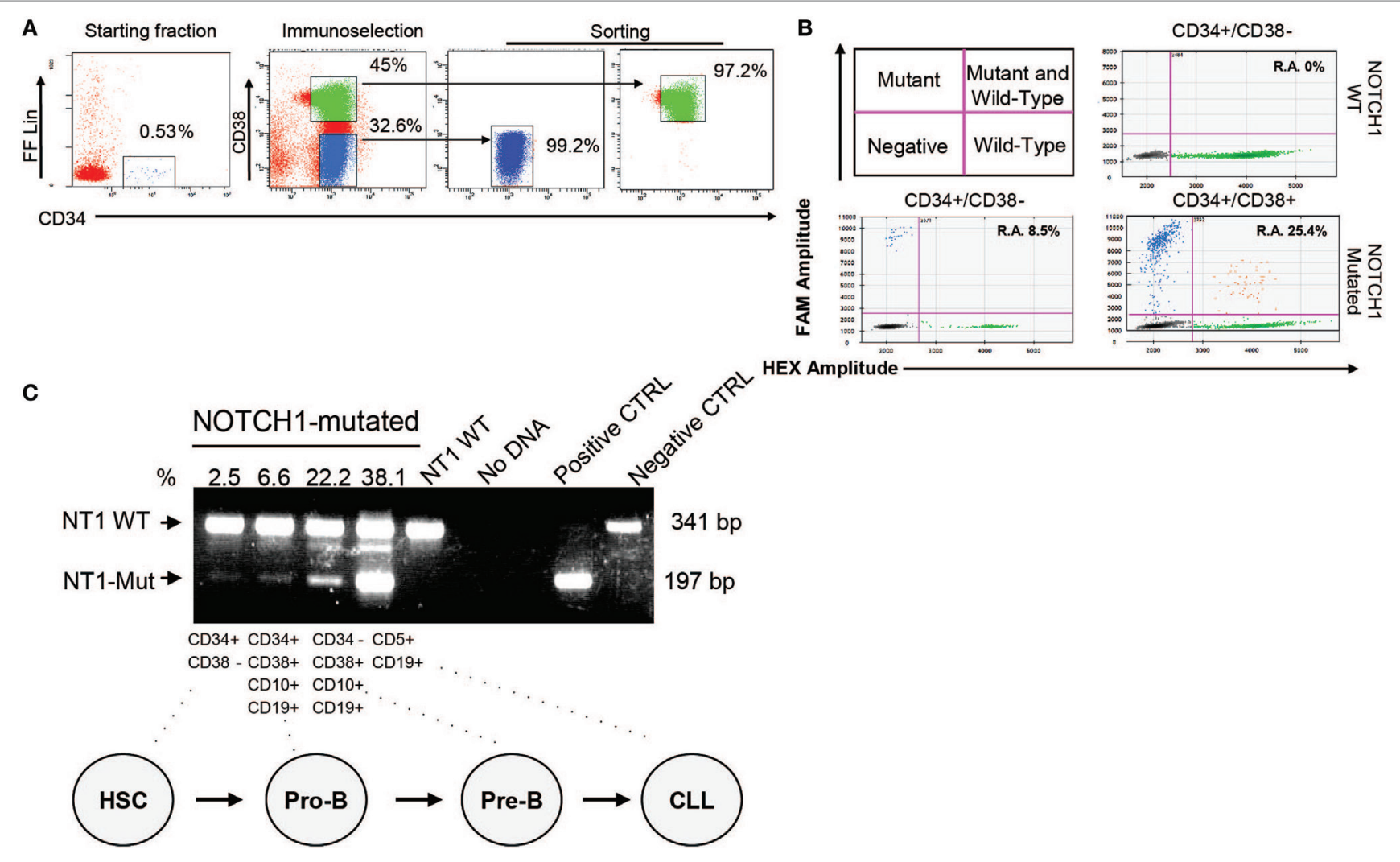

D
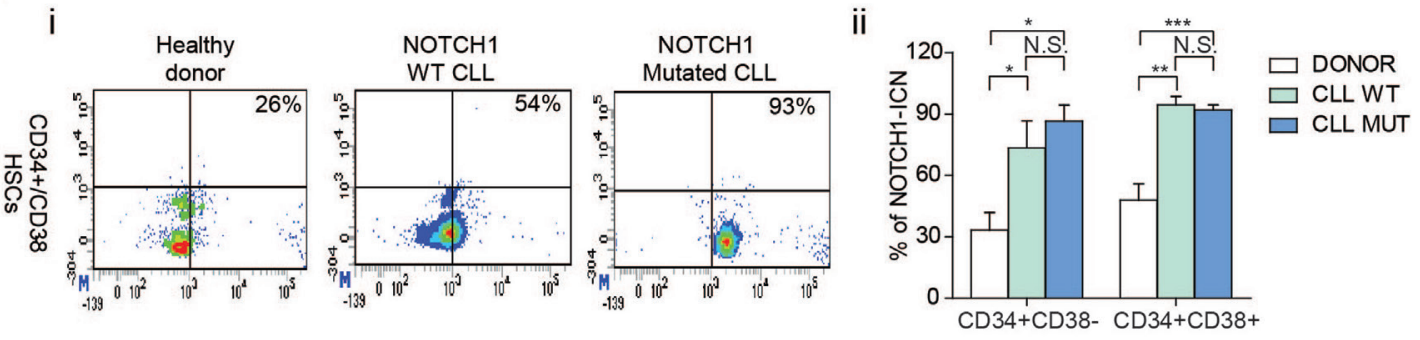

iii
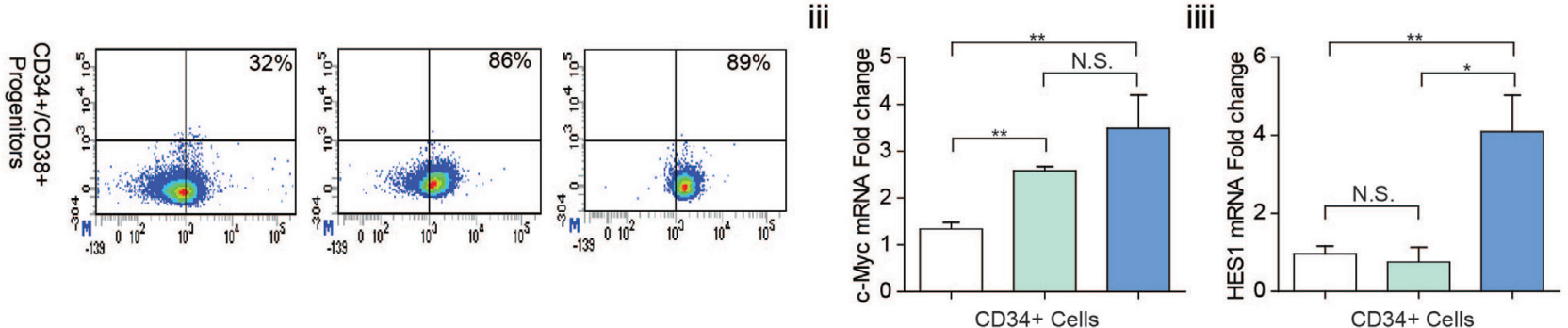

FIGURE 1 | Analysis of NOTCH1 gene mutation and signaling in bone marrow (BM) hematopoietic stem cells (HSCs) and progenitors cells. (A) FACS-setting used for HSCs and progenitor cells sorting purification of one representative NOTCH1-mutated patient. Prospective cell separation included immunoselection (middle plot) and sorting (right plot) to ensure purity and lack of chronic lymphocytic leukemia (CLL) cell contamination for NOTCH1 mutation analysis. (B) Droplet digital PCR. Upper left panel is a schematic representation of positive and negative droplet distribution according to the fluorophore threshold indicated in magenta lines. Upper right and lower panels are representative scatterplots of wild type and NOTCH1 mutated HSCs and progenitor cells, respectively. (C) Results of the allele-specific PCR assay for delCT NOTCH1 mutation in HSCs (CD34+CD38-), pro-B progenitors (CD34+CD38+CD10+CD19+), pre-B cells (CD34-CD38+CD10+CD19+), and B neoplastic CLL (CD5+CD19+) from one NOTCH1-mutated CLL sample. CD5+CD19+ cells from a NOTCH1-WT patient were used as negative control and showed a normal band of $341 \mathrm{bp}$. Samples bearing the delCT NOTCH1 mutation showed an additional mutant band of 197 bp. (D) (i) Representative dot plots of healthy control, NOTCH1 wild type, and NOTCH1 mutated CLL BM samples showing expression of NOTCH1-ICN on CD34+/ CD38- HSCs and CD34+/CD38+ progenitors compartments. (ii) Bar graphs show the means \pm SD of the percentage of NOTCH1-ICN positive cells. ${ }^{\star} p<0.05$, ${ }^{* *} p<0.01,{ }^{* *} p<0.001$ according to Student's $t$-test; (iii) real-time PCR analysis of c-MYC and Hes1 gene expression in CD34+ BM cells. mRNA levels were normalized to GAPDH and represented as fold change using healthy control cells as a reference. 


\section{HSCs From CLL Patients Have NOTCH1 Aberrantly Activated Also in Unmutated NOTCH1 Patients}

Thus, we analyzed the NOTCH1 signaling status in HSCs and progenitor cells of NOTCH1-mutated and unmutated CLL samples. Physiologically, the active intracellular domain (ICN) of NOTCH1 accumulates in cells with activated NOTCH1 signaling as a result of a cleavage of the transmembrane (TM) subunit made by the y-secretase complex (14). Here, we used flow cytometry to quantitate the percentage of active NOTCH1-ICN in CD34+/ CD38- HSCs and CD34+CD38+ progenitors from the BM of CLL patients and HDs, used as control. As shown in Figure 1Di,ii, NOTCH1-ICN was significantly higher in CLL samples regardless the NOTCH1 mutational status compared to non-leukemic samples. Indeed, the mean percentage of CD34+/CD38-/ NOTCH1-ICN+ and CD34+/CD38+/NOTCH1-ICN+ populations in NOTCH1 WT and mutated CLL was significantly higher than HDs samples $(73.4 \pm 22.9$ and $83 \pm 16.4$ vs $33.3 \pm 14.8 \%$; $94.4 \pm 7.3$ and $92.8 \pm 4.3$ vs $47.9 \pm 13.8 \%$, respectively).

To demonstrate the capability of CD34+ CLL cells to activate NOTCH1 signaling pathway, we analyzed the levels of NOTCH1 downstream transcriptional target gene. It has been demonstrated that NOTCH1 controls c-MYC expression in mature CLL cells overexpressing the NOTCH1-ICN (12). Thus, using quantitative reverse transcription-PCR, we found significantly higher mRNA expression levels of c-MYC in CD34+ cells from NOTCH1 mutated and WT CLL samples compared to HD $(3.5 \pm 0.7$ and $2.6 \pm 0.08$ vs $1.3 \pm 0.1$ ) (Figure 1Diii). In addition, we showed higher Hes1 expression in CD34 + cells from CLL patients compared to HD $(4.2 \pm 1.1$ vs $1.1 \pm 0.2)$ (Figure 1Diii), in line with upregulated NOTCH1 pathway.

Next, we analyzed whether the higher levels of NOTCH1 activation in HSCs and progenitors CLL samples were accompanied with increased NOTCH1 expression. We analyzed by western blot the expression levels of NOTCH1-TM subunit in CD34+ cells from BM aspirates of four NOTCH1-mutated CLL patients and three HDs (6). The median purity of immunoselected CD34+ cells was 97.3\% (range 74-99.5\%; Figure 2A). Results revealed that CD34+ samples from CLL patients always expressed the NOTCH1-TM protein. Conversely, in CD34+ cells from HDs, NOTCH1-TM was either absent or expressed at lower levels than those observed in CLL samples (Figure 2Bi,ii). These data demonstrated that high levels of NOTCH1 signaling activation correlated with NOTCH1-TM overexpression in the CD34+ hematopoietic compartment of CLL.

\section{DISCUSSION}

The Notch pathway is genetically altered in a large number of hematopoietic and solid tumors (15). We recently reported that activating mutations of NOTCH1 are recurrently associated with CLL and predict poor outcome $(3,4)$. The identification of a somatic NOTCH1 mutation could help providing additional information on the cellular origin of CLL. Sanger sequencing analysis (13) failed to detect mutations in CD34+/CD38- HSCs fractions of CLL patients. However, the sensitivity of this method does not allow the identification of a mutation whose allelic representation is $<10 \%$. Thus, we used more sensitive PCR based methods in order to verify results obtained with direct sequencing.

The analysis of CD34+/CD38+ progenitors detected the NOTCH1 mutation in $57 \%$ of the samples. Damm et al. (8) described a similar frequency of NOTCH1 mutation in multipotent progenitors of CLL samples performing NGS analysis on rare CD34+CD19- peripheral blood cells. Recently, the same analysis was conducted in immunoselected CD34+ BM cells that resulted NOTCH1-mutated in 8 out of 13 CLL samples (9). Here, we used two high sensitive PCR assays specific for NOTCH1 mutation and employed FACS sorted CD34+CD38+ cells from $\mathrm{BM}$ aspirates. Additionally, we showed here that the NOTCH1 mutational burden increased along specific stages of HSC differentiation in NOTCH1-mutated CLL patients. This suggests that the NOTCH1 lesion is selected and expands during HSC differentiation toward a $\mathrm{B}$ neoplastic cell, thus strengthening the hypothesis that the genetic alteration is an initial event associated with the stepwise malignant transformation of CLL.

In normal human BM, CD34+CD38- populations do not rearrange the IgH gene (16), thus raising issues on the molecular purity of the sorted hematopoietic cell fractions presenting a VDJ rearrangement. Nevertheless, the high purity of HSC doublesorted samples together with the sensitivity of the $\mathrm{IgH}$ method (5\%) limited the risk of detecting small fractions of contaminating neoplastic B cells. The alternative option is that $\mathrm{IgH}$ rearrangement is the expression of a B neoplastic transactivation at the HSC level.

Recently, common nonmutational NOTCH1 activation has been described in mature CLL cells (12) raising the question of whether the same condition is present in HSCs to determine their aberrant behavior. The Notch1 signaling has been extensively analyzed in the contest of embryonic hematopoiesis. Ex vivo approaches suggest that Notch signaling can expand HSCs, raising the question of whether this is a physiologic Notch function. Gerhardt et al. (17) correlated NOTCH1 with hematopoiesis in animal models and identified cell-autonomous functions for Notch1 signaling in fetal HSCs homeostasis. The present study indicated that the pool of CD34+ cells, including HSC and progenitor compartments, tend to have NOTCH1 aberrantly expressed and activated in CLL patients compared to HDs. NOTCH1 deregulation and overepression of c-Myc are independent of NOTCH1 mutational status. These data clearly show that expansion of the leukemic stem cell clone does not necessarily require a mutation to upregulate the $\mathrm{NOTCH} 1$ signaling, suggesting the presence of extrinsic factors from the BM HSC niche that are capable of stimulating and promoting CLL-initiating cell clone expansion. In CLL patients, BM mesenchimal cells express different ligands, which might play a role in NOTCH1 activation (18). However, additional studies are warranted to compare the levels and type of these ligands in the BM of healthy people vs CLL patients. Alternatively, CLL-HSCs may have cell-intrinsic mechanisms activating NOTCH1, which involve alterations of NOTCH1 pathway regulators $(19,20)$ or aberrant regulation of NOTCH1 receptor recycling (21). This selective pressure might contribute to the onset of specific NOTCH1 mutations in a DNA context that is prone to spontaneous microdeletion (5) (Figure 2C). 

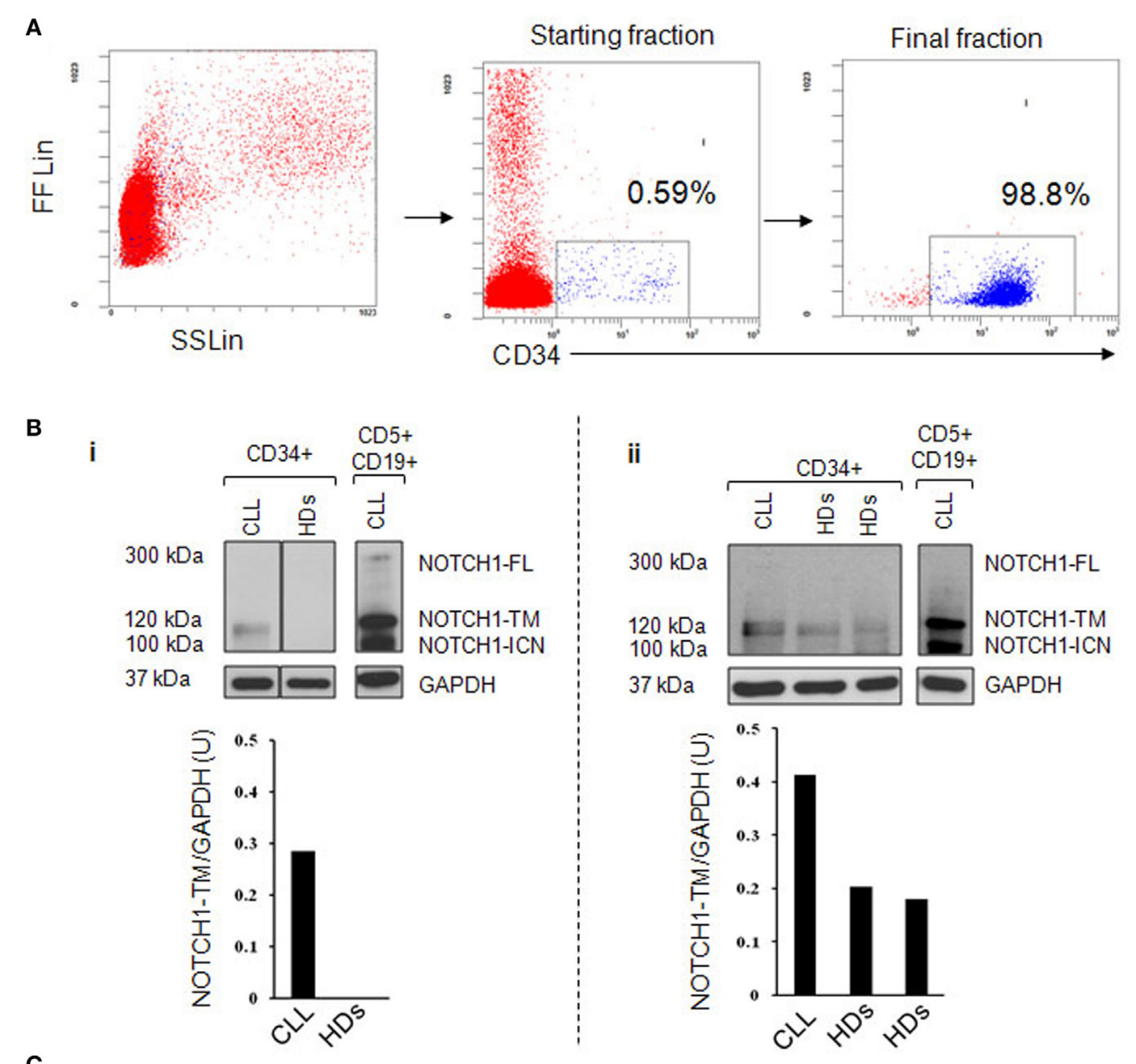

C

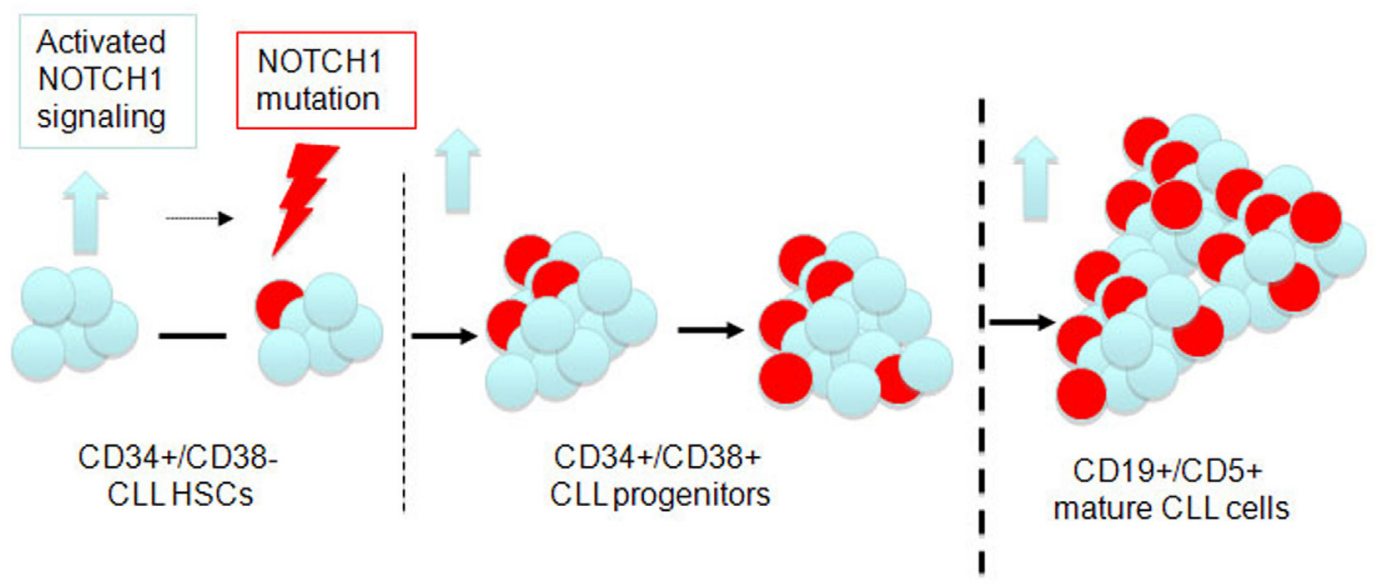

FIGURE 2 | Analysis of NOTCH1-TM expression in immunoselected CD34+ bone marrow (BM) cells. (A) Immunomagnetic cell sorting purification and phenotypic characterization of CD34+ cells from a representative chronic lymphocytic leukemia (CLL) BM used for NOTCH1 protein expression analysis. (B) (i and ii) Western blot analysis. Vertical lines indicate realignment of the same blot imaging. Protein loading was assessed by reprobing the blots with an anti-GAPDH antibody. The density of the bands corresponding to NOTCH1-TM was evaluated by densitometric analysis. Densitometry units (U) were calculated relative to GAPDH. Results revealed that CD34+ samples from CLL patients always expressed the NOTCH1-TM protein. (C) Schematic representation of clonal evolution during the development of CLL starting from hematopoietic stem cells (HSCs). NOTCH1 is found to be active at early stage of hematopoiesis (blue arrow) together with NOTCH1 mutations (lightning arrow) to which activated signaling might contribute. NOTCH1 activation persists and the mutated clone expands as the cell commit to mature CD19+CD5+CLL. 
Our discovery of NOTCH1 deregulated signal and mutations in CLL-HSC have significant therapeutic implications in this disease. A variety of approaches was used to inhibit NOTCH1 for cancer therapy, including presenilin $\gamma$-secretasi inhibtors, trafficking modulators (22), and blocking antibodies. In the next future, it will be interesting to evaluate the effects of these anti-NOTCH1 drugs in the development of CD34+CD38- and CD34+CD38+ CLL populations.

In conclusion, our data confirmed the presence of NOTCH1 mutations in HSCs of CLL patients and showed for the first time a common nonmutational NOTCH1 activation occurring early in CLL hematopoiesis and represent a rationale for the use of therapies targeting the NOTCH1 signaling in CLL aimed to inhibit the survival of CLL-initiating cells.

\section{ETHICS STATEMENT}

This study was carried out in accordance with the recommendations of University/Hospital of Perugia guidelines, Ethics Committee of Perugia, with written informed consent from all subjects. All subjects gave written informed consent in accordance with the Declaration of Helsinki. The protocol was approved by the Ethics Committee of Perugia.

\section{REFERENCES}

1. Chiorazzi N, Ferrarini M. Cellular origin(s) of chronic lymphocytic leukemia: cautionary notes and additional considerations and possibilities. Br J Haematol (2011) 117:1781-91. doi:10.1182/blood-2010-07-155663

2. Kikushige Y, Ishikawa F, Miyamoto T, Shima T, Urata S, Yoshimoto G, et al. Self-renewing hematopoietic stem cell is the primary target in pathogenesis of human chronic lymphocytic leukemia. Cancer Cell (2011) 20:246-59. doi:10.1016/j.ccr.2011.06.029

3. Di Ianni M, Baldoni S, Rosati E, Ciurnelli R, Cavalli L, Martelli MF, et al. A new genetic lesion in B-CLL: a NOTCH1 PEST domain mutation. Br J Haematol (2009) 146:689-91. doi:10.1111/j.1365-2141.2009.07816.x

4. Sportoletti P, Baldoni S, Cavalli L, Del Papa B, Bonifacio E, Ciurnelli R, et al. NOTCH1 PEST domain mutation is an adverse prognostic factor in B-CLL. Br J Haematol (2010) 151:404-6. doi:10.1111/j.1365-2141.2010.08368.x

5. South AP, Cho RJ, Aster JC. The double-edged sword of Notch signaling in cancer. Sem Cell Dev Biol (2012) 23:458-64. doi:10.1016/j.semcdb.2012.01.017

6. Rosati E, Sabatini R, Rampino G, Tabilio A, Di Ianni M, Fettucciari K, et al. Constitutively activated Notch signaling is involved in survival and apoptosis resistance of B-CLL cells. Blood (2009) 113:856-65. doi:10.1182/ blood-2008-02-139725

7. De Falco F, Sabatini R, Del Papa B, Falzetti F, Di Ianni M, Sportoletti P, et al. Notch signaling sustains the expression of Mcl-1 and the activity of eIF4E to promote cell survival in CLL. Oncotarget (2015) 6:16559-72. doi:10.18632/ oncotarget. 4116

8. Damm F, Mylonas E, Cosson A, Yoshida K, Della Valle V, Mouly E, et al. Acquired initiating mutations in early hematopoietic cells of CLL patients. Cancer Discov (2014) 4:1088-101. doi:10.1158/2159-8290.CD-14-0104

9. Quijada-Álamo M, Hernández-Sánchez M, Robledo C, Hernández-Sánchez JM, Benito R, Montaño A, et al. Next-generation sequencing and FISH studies reveal the appearance of gene mutations and chromosomal abnormalities in hematopoietic progenitors in chronic lymphocytic leukemia. J Hematol Oncol (2017) 10:83. doi:10.1186/s13045-017-0450-y

10. Rossi FM, Zucchetto A, Tissino E, Dal Bo M, Bomben R, Caldana C, et al. CD49d expression identifies a chronic-lymphocytic leukemia subset with high levels of mobilized circulating CD34(+) hemopoietic progenitors cells. Leukemia (2014) 28:705-8. doi:10.1038/leu.2013.331

\section{AUTHOR CONTRIBUTIONS}

MDI and PS designed experiments; SB, BDP, PA, ED, FDF, ADT, RG, PA, GR, CR, ESB, and AFC performed experiments and analyzed data; EA and FF contributed to samples collection; MDI, ER, BDP, IS, and PS wrote the manuscript.

\section{FUNDING}

This study was supported by grants from MIUR (Scientific Independence of young Researchers 2014 Grant No. RBSI14GPBL to PS and Progetti di Ricerca di Interesse Nazionale 2010-2011 Grant No. 2010MCLPLB to IS), AIRC (My First AIRC Grant No. 17442 to PS), the Gilead Fellowship Program 2017 to PS. "Associazione Umbra Leucemie e Linfomi," Perugia, Italy; "Associazione Italiana Leucemie, Linfomi e Mieloma," LAquila Section, Italy.

\section{SUPPLEMENTARY MATERIAL}

The Supplementary Material for this article can be found online at https://www.frontiersin.org/articles/10.3389/fonc.2018.00105/ full\#supplementary-material.

11. Kluk MJ, Ashworth T, Wang H, Knoechel B, Mason EF, Morgan EA, et al. Gauging NOTCH1 activation in cancer using immunohistochemistry. PLoS One (2013) 8:e67306. doi:10.1371/journal.pone.0067306

12. Fabbri G, Holmes AB, Viganotti M, Scuoppo C, Belver L, Herranz D, et al. Common nonmutational NOTCH1 activation in chronic lymphocytic leukemia. Proc Natl Acad Sci U S A (2017) 114:E2911-9. doi:10.1073/ pnas. 1702564114

13. Sportoletti P, Baldoni S, Del Papa B, Aureli P, Dorillo E, Ruggeri L, et al. A revised NOTCH1 mutation frequency still impacts survival while the allele burden predicts early progression in chronic lymphocytic leukemia. Leukemia (2014) 28:436-9. doi:10.1038/leu.2013.289

14. De Falco F, Sabatini R, Falzetti F, Di Ianni M, Sportoletti P, Baldoni S, et al. Constitutive phosphorylation of the active Notchl intracellular domain in chronic lymphocytic leukemia cells with NOTCH1 mutation. Leukemia (2015) 29:994-8. doi:10.1038/leu.2014.329

15. Ntziachristos P, Lim JS, Sage J, Aifantis I. From fly wings to targeted cancer therapies: a centennial for notch signaling. Cancer Cell (2014) 25:318-34. doi:10.1016/j.ccr.2014.02.018

16. Davi F, Faili A, Gritti C, Blanc C, Laurent S, Sutton L, et al. Early onset of immunoglobulin heavy chain gene rearrangments in normal human bone marrow CD34+ cells. Blood (1997) 90:4014-21.

17. Gerhardt DM, Pajcini KV, D’Altri T, Tu L, Jain R, Xu L, et al. The Notch1 transcriptional activation domain is required for development and reveals a novel role for Notchl signaling in fetal hematopoietic stem cells. Genes Dev (2014) 28:576-93. doi:10.1101/gad.227496.113

18. Nwabo Kamdje AH, Bassi G, Pacelli L, Malpeli G, Amati E, Nichele I, et al. Role of stromal cell-mediated notch signaling in CLL resistance to chemotherapy. Blood Cancer J (2012) 2(5):e73. doi:10.1038/bcj.2012.17

19. Baldoni S, Sportoletti P, Del Papa B, Aureli P, Dorillo E, Rosati E, et al. NOTCH and NF- $\mathrm{\kappa B}$ interplay in chronic lymphocytic leukemia is independent of genetic lesion. Int J Hematol (2013) 98:153-7. doi:10.1007/s12185-013-1368-y

20. PuenteXS, BeàS, Valdés-MasR, VillamorN, Gutiérrez-AbrilJ,Martin-SuberoJI, et al. Non-coding recurrent mutations in chronic lymphocytic leukaemia. Nature (2015) 526:519-24. doi:10.1038/nature14666

21. Le Bras S, Loyer N, Le Borgne R. The multiple facets of ubiquitination in the regulation of notch signaling pathway. Traffic (2011) 12:149-61. doi:10.1111/j.1600-0854.2010.01126.x 
22. Baldoni S, Del Papa B, Dorillo E, Aureli P, De Falco F, Rompietti C, et al. Bepridil exhibits anti-leukemic activity associated with NOTCH1 pathway inhibition in chronic lymphocytic leukemia. Int J Cancer (2018). doi:10.1002/ ijc.31355

Conflict of Interest Statement: The authors declare that the research was conducted in the absence of any commercial or financial relationships that could be construed as a potential conflict of interest.
Copyright (C) 2018 Di Ianni, Baldoni, Del Papa, Aureli, Dorillo, De Falco, Albi, Varasano, Di Tommaso, Giancola, Accorsi, Rotta, Rompietti, Silva Barcelos, Campese, Di Bartolomeo, Screpanti, Rosati, Falzetti and Sportoletti. This is an open-access article distributed under the terms of the Creative Commons Attribution License (CC BY). The use, distribution or reproduction in other forums is permitted, provided the original author(s) and the copyright owner are credited and that the original publication in this journal is cited, in accordance with accepted academic practice. No use, distribution or reproduction is permitted which does not comply with these terms. 